Methods This study uses a random nationally-representative sample of 4111 adults aged 25-64 years (78\% response rate) collected in 2010 using the WHO STEPwise survey methods, restricted to non-pregnant participants with valid weight and height measurements $(n=3533)$. We categorised body mass index from measured height and weight to determine underweight, overweight and obesity (WHO thresholds). Analyses were stratified by gender. All analyses were weighted for nonresponse and adjusted for complex survey design using STATA14. We conducted multivariate multinomial regression analysis to identify factors associated with underweight, overweight and obesity, using normal weight as reference. Fully adjusted relative risk ratios (ARRR) with their corresponding 95\% confidence intervals (CI) are reported.

Results Two-fifths of adults in The Gambia were overweight or obese, with a higher prevalence of obesity in women (17.0\%, [95\% CI $14.7 \%$ to $19.7 \%$ ] vs $8.1 \%$ in men, [6.0$11.0 \%]$ ) and urban residents. Urban residence, abdominal obesity, higher education, and age were significantly associated with obesity among both men and women. Obesity was also significantly associated with low fruit and vegetable intake in men, and with hypertension and ethnicity in women. Most of these variables were also significantly associated with overweight. Compared with rural residents, the risk of overweight and obesity among urban residents were three- and six-fold higher respectively in men (overweight: ARRR 3.1, 95\% CI 1.7 to 5.6 ; obesity: $6.6,2.5-17.2$ ) and in women (overweight: 3.2, 1.9-5.4; obesity: 5.9, 3.1-11.2). No significant associations were found for underweight, except for smoking and ethnicity in men and old age and ethnicity in women.

Conclusion This study reveals that the burden of obesity is increasing at an alarming rate in The Gambia. Preventive strategies should be directed at raising awareness of the risk factors, discouraging harmful beliefs on weight, and the promotion of healthy diet and physical activity particularly in urban areas and among women.

\section{P48 WHAT BARRIERS AND FACILITATORS INFLUENCE THE IMPLEMENTATION OF NEW HIGH-RISK MEDICINE SERVICES IN SCOTTISH COMMUNITY PHARMACIES?}

${ }^{1} \mathrm{NM}$ Weir*, ${ }^{1} \mathrm{R}$ Newham, ${ }^{1} \mathrm{E}$ Dunlop Corcoran, ${ }^{1,2} \mathrm{M}$ Bennie. 'Strathclyde Institue of Pharmacy and Biomedical Sciences, University of Strathclyde, Glasgow, UK; ${ }^{2}$ Public Health and Intelligence, National Services Scotland, Edinburgh, UK

\subsection{6/jech-2018-SSMabstracts.173}

Background In the UK, $6.5 \%$ of hospital admissions are attributed to adverse effects of high-risk medicines, including warfarin and non-steroidal anti-inflammatory drugs (NSAIDs). Warfarin and NSAIDs 'care bundles' were developed to address this. These services focus on improving care processes and patient outcomes. They focus on medication-specific interactions and patient education, and were piloted in 2017 in 24 pharmacies across four healthcare regions, with intent for national implementation. This study aims to identify barriers and facilitators influencing their successful implementation.

Methods Following a systematic review identifying barriers and facilitators to the national implementation of community pharmacy innovations, a questionnaire was developed and disseminated in June 2017 to pharmacy staff $(n=217)$ in the pilot pharmacies. Multivariate regression analysis was conducted to identify barriers and facilitators influencing successful implementation (determined using a 6-item Likert-scale). Pearsons-
Chi Square test was used to identify differences between the bundles.

Results Seventy-four participants (34.1\%) responded from 17 pharmacies (70.8\%). $73.1 \%$ and $72.1 \%$ considered the warfarin and NSAIDs bundles successfully implemented respectively. Multivariate regression analysis identified two factors influencing successful implementation of the warfarin bundle $\left(\mathrm{p}<0.001, \mathrm{R}^{2}=0.752\right)$ : compatibility of the bundle with pharmacies' processes; and positive perceptions of patients. For the NSAIDs bundle, four predictors of implementation success $\left(\mathrm{p}<0.001, \mathrm{R}^{2}=0.633\right)$ were identified: pharmacy staff having sufficient knowledge of NSAIDs; perceived incentives involved in bundle delivery; workload of the NSAIDs bundle; and funding. Compared to the NSAIDs bundle, pharmacy staff involved with the warfarin bundle were less likely to agree there were sufficient pharmacy staff $(p=0.030)$, and that they had enough time $(p=0.025)$ and training $(p=0.004)$ to deliver the bundle.

Conclusion The questionnaire successfully identified barriers and facilitators to the implementation of the bundles. Warfarin bundle success factors (compatibility and positive patient perceptions) were unsurprising as previous strategies in Scotland have engaged pharmacies with warfarin counselling, and patients are likely to have experienced some warfarin counselling already. This is not the case for NSAIDs, therefore it is understandable that additional strategic facilitators (incentives and funding) were success factors. Workload associated with the NSAIDs bundle was a risk to successful implementation, potentially explained by the greater number of patients from over-the-counter purchases and prescription supplies. Contextual factors not captured may explain differences (e.g. regional staffing issues may explain why warfarin bundle participants were less likely to think they had sufficient staff and time).

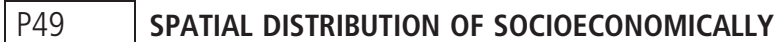 DEPRIVED IMMIGRANTS AND THEIR ACCESS TO HEALTHCARE SERVICES IN A NORTHERN CITY IN CHILE}

${ }^{1} \mathrm{~A}$ Vasquez*, ${ }^{2} \mathrm{~B}$ Cabieses, ${ }^{3} \mathrm{~K}$ Tudesca. ${ }^{1}$ School of Geosciences, University of Edinburgh, Edinburgh, UK; ${ }^{2}$ Faculty of Medicine, Universidad del Desarrollo, Santiago, Chile; ${ }^{3}$ Institute of Geography, Pontificia Universidad Catolica de Chile, Santiago, Chile

\subsection{6/jech-2018-SSMabstracts. 174}

Background Immigrant population have exponentialy grown in the past thirty years in Chile, concentrating in the northern and central areas of the country. Iquique is one of the densest cities in the country in terms of international migrants, reaching around $10 \%$ of total population. In this city, healthcare network is divided into 4 primary care centres and one hospital. No study; however, has analysed where socioeconomically deprived immigrants are located in the city and how they access healthcare services compared to the local population. This study aimed at analysing the spatial location of international migrants in socioeconomic deprivation in the city of Iquique and how they access public healthcare, as well as compare it to the locals.

Methods Secondary data analysis of 2012 census data in Chile. We built a multiple index of deprivation for immigrants and locals separately, with the following dimensions at the census track level $(n=1,879)$ : (i) educational level (none/primary/secondary/higher), (ii) any disability (yes/no), (iii) housing (overcrowded, collective, rented), (v) crime (theft/burglary) (iv) environment (traffic accidents). Dimensions of the index were 